\title{
Cytomegaloviral Infection
}

National Cancer Institute

\section{Source}

National Cancer Institute. Cytomegaloviral Infection. NCI Thesaurus. Code C53649.

A herpesvirus infection caused by Cytomegalovirus. Healthy individuals generally do not produce symptoms. However, the infection may be life-threatening in affected immunocompromised patients. The virus may cause retinitis, esophagitis, gastritis, and colitis. Morphologically, it is characterized by the presence of intranuclear inclusion bodies. 